\title{
SET PARTITIONS WITH SUCCESSIONS AND SEPARATIONS
}

AUGUSTINE O. MUNAGI

Received 31 August 2004

Partitions of the set $\{1,2, \ldots, n\}$ are classified as having successions if a block contains consecutive integers, and separated otherwise. This paper constructs enumeration formulas for such set partitions and some variations using Stirling numbers of the second kind.

\section{Introduction}

The number of ways of partitioning a set of $m$ elements into $k$ nonempty subsets (called classes or blocks) is given by $s 2(m, k)$, the Stirling number of the second kind. Without loss of generality we assume that the $m$ elements have been labeled $1,2, \ldots, m$ and consider $k$-partitions of the set $[m]=\{1,2, \ldots, m\}$. Substantial information on set partitions can be found in $[3,8]$. For connections of set partitions with the combinatorics of distributions and occupancy, to which the objects considered in this paper are also related, see $[2,5]$. Essential properties of the numbers $s 2(m, k)$ can be found in $[3,7]$.

Definition 1.1. A partition of $[m]$ is said to be $t$-separated $(t \geq 1)$ if the difference between every pair of integers in each class exceeds $t-1$ in absolute value. Denote the set of $t$-separated $k$-partitions of $[m]$ by $H^{t}(m, k)$, and let $h^{t}(m, k)$ represent the cardinality $\left|H^{t}(m, k)\right|$ of $H^{t}(m, k)$.

It follows that $h^{1}(m, k)=s 2(m, k)$. For example, members of $H^{3}(10,4)$ include $\{1,4,7\}$ $\{2,5,8\}\{3,6,9\}\{10\},\{1,4,7,10\}\{2,6,9\}\{3\}\{5,8\}$, and $\{1,4,8\}\{2,5,9\}\{3,7\}\{6,10\}$.

Separated combinations of elements of $[m]$ have been considered in [2, page 65], [5, page 198], [8, page 20], and [9, page 26]. Our terminology is adapted from [2, page 65]. The set $H^{2}(m, k)$, which can also be described as the set of nonconsecutive partitions of $[m]$, has already found an application in the enumeration of complementing systems of subsets of $\{0,1, \ldots, m-1\}$ (see $[4]$ ).

Partitions with successions, which we define next, give another generalization of $H^{2}(m, k)$, but in the opposite direction. The associated theory is relatively complicated. We give the definition of partitions with pairwise successions here, and consider the general case in Section 4. 
Definition 1.2. A partition of $[m]$ is said to have $r$ successions $(r \geq 0)$ if it contains $r$ pairs of consecutive integers, where each pair of consecutive integers is counted within one class, and a string of more than two consecutive integers in a class are considered two at a time. The set of $k$-partitions of $[m]$ with $r$ successions will be denoted by $C_{r}(m, k)$.

Thus $C_{0}(m, k)=H^{2}(m, k)$. For example, members of $C_{3}(10,4)$ include $\{1,2\}\{3,4,7\}$ $\{5,6,9\}\{8,10\},\{1,2,3,5\}\{4,6,7\}\{8,10\}\{9\}$, and $\{1,2,3,4\}\{5,7,9\}\{6,8\}\{10\}$.

Our terminology is consistent with that of $[6$, page 11] which deals with combinations of elements of $[\mathrm{m}]$ with a prescribed number of successions. We define a distinguished subset of $C_{r}(m, k)$.

Definition 1.3. Let $p \in C_{r}(m, k)$. The $r$ successions in $p$ will be called detached if $p$ contains no $t$-string of consecutive integers, where $t>2$. Denote the set of partitions of $[m]$ with $r$-detached successions by $D_{r}(m, k)$.

For example, members of $D_{3}(10,4)$ include $\{1,2\}\{3,4,7\}\{5,6,9\}\{8,10\}$ and $\{1,2,4,5\}$ $\{3,6,7\}\{8,10\}\{9\}$. Note that $\{1,2,3,5\}\{4,6,7\}\{8,10\}\{9\}$ belongs to $C_{3}(10,4)$ but not to $D_{3}(10,4)$ because the two successions in $\{1,2,3,5\}$ are not detached.

Let $c_{r}(m, k)=\left|C_{r}(m, k)\right|$ be the cardinality of $C_{r}(m, k)$. Similarly let $d_{r}(m, k)=$ $\left|D_{r}(m, k)\right|$. Then $c_{r}(m, k)=d_{r}(m, k)$ for $r=0,1$. The number $c_{r}(m, k)$ bears a kind of duality relationship with $d_{r}(m, k)$, as shown in the next section.

Remark 1.4. Expectedly the set difference $E_{r}(m, k)=C_{r}(m, k)-D_{r}(m, k)$ consists of partitions of $[m]$ with $r$-nondetached successions, that is, partitions in which at least a class in each partition contains a string of three or more consecutive integers, provided $r>1$. As usual, let $e_{r}(m, k)=\left|E_{r}(m, k)\right|$. Hence $e_{r}(m, k)=c_{r}(m, k)-d_{r}(m, k)$.

Remark 1.5. The related category of partitions of $[m]$ in which every class consists of consecutive integers is already well known. They correspond to the compositions of $m$ into $k$ parts [1, page 55] and their number $c(m, k)$ is given by the simple formula

$$
c(m, k)=\left(\begin{array}{c}
m-1 \\
k-1
\end{array}\right) \Longrightarrow c(m) \sum_{k} c(m, k)=2^{m-1} .
$$

The next two sections are devoted to statements and proofs of essential results. The last section examines certain generalizations of them.

\section{Statement of results}

The first theorem gives the recurrence equation satisfied by the number $h^{t}(m, k)$ of $t$ separated $k$-partitions of $[m]$, and its solution.

Theorem 2.1. (i) $h^{t}(m, k)=h^{t}(m-1, k-1)+(k-t+1) h^{t}(m-1, k), h^{t}(m, t)=1,1 \leq$ $t \leq k \leq m$.

(ii) $h^{t}(m, k)=s 2(m-t+1, k-t+1)$.

(iii) $h^{t}(m)=\sum_{k} h^{t}(m, k)=B(m-t+1)$, where $B(m)$ denotes the $m t h$ Bell number. 
Theorem 2.1(ii) implies the following relation for $h^{t}(m, k)$ :

$$
h^{t}(m, k)=h^{t+j}(m+j, k+j), \quad j=0, \pm 1, \pm 2, \ldots .
$$

Thus in particular, $h^{t}(m, k)=h^{t-1}(m-1, k-1)$,

$$
h^{2}(m, k)=h^{1}(m-1, k-1)=s 2(m-1, k-1)=c_{0}(m, k),
$$

where the last equality follows from Definition 1.2. This implies

$$
h^{t}(m, k)=h^{2}(m-t+2, k-t+2)=c_{0}(m-t+2, k-t+2) .
$$

TheOREM 2.2. The number $c_{r}(m, k)$ of $k$-partitions of $[m]$ with $r$ successions satisfies the recurrence

$$
\begin{array}{r}
c_{r}(m, k)=c_{r}(m-1, k-1)+(k-1) c_{r}(m-1, k)+c_{r-1}(m-1, k), \\
0 \leq r \leq m-k, \quad 1 \leq k \leq m-r, \quad c_{0}(m, k)=s 2(m-1, k-1) .
\end{array}
$$

The following theorem gives the solution of (2.4).

Theorem 2.3.

$$
c_{r}(m, k)=\left(\begin{array}{c}
m-1 \\
r
\end{array}\right) s 2(m-r-1, k-1) .
$$

Remark 2.4. Theorem 2.3 leads to the expected fact that $\sum_{r \geq 0} c_{r}(m, k)=s 2(m, k)$, the verification of which is immediate since it coincides with a standard identity, see, for example, [5, page 43]. In particular, $\sum_{r \geq 0} c_{r}(m, 2)=\sum_{0 \leq r \leq m-2}\left(\begin{array}{c}m-1 \\ r\end{array}\right)=2^{m-1}-1=s 2(m, 2)$.

By means of (2.4) and (2.5) we derive the following additional results for $c_{r}(m, k)$.

(i)

$$
\left(\begin{array}{c}
m-2 \\
r-1
\end{array}\right) c_{r}(m, k)=\left(\begin{array}{c}
m-1 \\
r
\end{array}\right) c_{r-1}(m-1, k) \quad \text { or } \quad c_{r}(m, k)=\frac{m-1}{r} c_{r-1}(m-1, k),
$$

which may be iterated to give

$$
c_{r}(m, k)=\frac{(m-1)_{j+1}}{(r)_{j+1}} c_{r-j-1}(m-j-1, k), \quad 0 \leq j<r,
$$

where $(m)_{k}$ is the falling factorial defined by $(m)_{k}=m(m-1) \cdots(m-k+1)$.

Therefore

$$
c_{r}(m, k)=\left(\begin{array}{c}
m-1 \\
r
\end{array}\right) c_{0}(m-r, k)
$$

Note that (2.8) can also be obtained directly from (2.5) since $c_{0}(m, k)=s 2(m-1$, $k-1)$. 
454 Set partitions with successions and separations

(ii)

$$
c_{r}(m, k)=\frac{m-1}{m-r-1}\left(c_{r}(m-1, k-1)+(k-1) c_{r}(m-1, k)\right) .
$$

Observe that (ii) implies (2.6).

The following corollary is immediate from Theorem 2.3.

Corollary 2.5. If $c_{r}(m)$ denotes the total number of partitions of $[m]$ with $r$ successions, then

$$
c_{r}(m)=\left(\begin{array}{c}
m-1 \\
r
\end{array}\right) B(m-r-1), \quad m \geq r+1 .
$$

Remark 2.6. Corollary 2.5 suggests the summation of (2.10) over $r$ to obtain the total number $B(m)$ of partitions of $[m]$. This leads to the recurrence for the Bell numbers as follows:

$$
B(m)=\sum_{r=0}^{m-1} c_{r}(m)=\sum_{r=0}^{m-1}\left(\begin{array}{c}
m-1 \\
r
\end{array}\right) B(m-r-1)=\sum_{r=0}^{m-1}\left(\begin{array}{c}
m-1 \\
m-r-1
\end{array}\right) B(m-r-1) .
$$

Thus by (2.11) we obtain a natural interpretation of the summands in the Bell recurrence [9, page 23], $\sum_{0 \leq j \leq m-1}\left(\begin{array}{c}m-1 \\ j\end{array}\right) B(j)$, as the distribution of the numbers of partitions of $[m]$ according to decreasing numbers $r$ of successions in a partition, $r=m-1, m-2, \ldots, 0$.

Theorem 2.7. The number $d_{r}(m, k)$ of $k$-partitions of $[m]$ with $r$-detached successions satisfies the recurrence

$$
\begin{aligned}
d_{r}(m, k)= & d_{r}(m-1, k-1)+(k-1) d_{r}(m-1, k)+d_{r-1}(m-2, k-1) \\
& +(k-1) d_{r-1}(m-2, k)
\end{aligned}
$$

$d_{0}(m, k)=s 2(m-1, k-1), 0 \leq r \leq\lfloor m / 2\rfloor, 1 \leq k \leq m-r$, where $\lfloor N\rfloor$ denotes the integer part of $N$, and the last equality in $1 \leq k \leq m-r$ requires $m \geq 2 r$.

The solution of (2.12) is given in the next theorem.

Theorem 2.8 .

$$
d_{r}(m, k)=\left(\begin{array}{c}
m-r \\
r
\end{array}\right) s 2(m-r-1, k-1), \quad 1 \leq k \leq m-r .
$$

Using (2.13) and previous results additional relations are obtained for $d_{r}(m, k)$.

(i) First, from (2.5) and (2.13), it is deduced that

$$
d_{r}(m, k)=\frac{(m-r)_{r}}{(m-1)_{r}} c_{r}(m, k)
$$


(ii) By using (2.14) in (2.6),

$$
d_{r}(m, k)=\frac{m-2 r+1}{r} d_{r-1}(m-1, k)
$$

which may be iterated to

$$
d_{r}(m, k)=\frac{(m-2 r+1)^{(j)}}{(r)_{j}} d_{r-j}(m-j, k), \quad 1 \leq j \leq r
$$

where $(m)^{(k)}$ is the rising factorial defined by $(m)^{(k)}=m(m+1) \cdots(m+k-1)$. Therefore

$$
d_{r}(m, k)=\left(\begin{array}{c}
m-r \\
r
\end{array}\right) d_{0}(m-r, k)
$$

(iii)

$$
d_{r}(m, k)=\frac{m-r}{m-2 r}\left(d_{r}(m-1, k-1)+(k-1) d_{r}(m-1, k)\right)
$$

or

$$
d_{r}(m, k)=\frac{m-r}{r}\left(d_{r-1}(m-2, k-1)+(k-1) d_{r-1}(m-2, k)\right) .
$$

The following corollary follows from Theorem 2.8.

Corollary 2.9. If $d_{r}(m)$ denotes the total number of partitions of $[m]$ with $r$-detached successions, then

$$
d_{r}(m)=\left(\begin{array}{c}
m-r \\
r
\end{array}\right) B(m-r-1), \quad m \geq r+1 .
$$

Hence, by (2.13),

$$
d_{r}(m)=\frac{(m-r)_{r}}{(m-1)_{r}} c_{r}(m)
$$

The following corollary relates to the number $e_{r}(m, k)=c_{r}(m, k)-d_{r}(m, k)$ (see Remark 1.4): (i) follows from (2.5) and (2.13), and (ii) from (2.10) and (2.20).

Corollary 2.10. (i) $e_{r}(m, k)=\left[\left(\begin{array}{c}m-1 \\ r\end{array}\right)-\left(\begin{array}{c}m-r \\ r\end{array}\right)\right] s 2(m-r-1, k-1)$.

(ii) $e_{r}(m)=\sum_{k} e_{r}(m, k)=\left[\left(\begin{array}{c}m-1 \\ r\end{array}\right)-\left(\begin{array}{c}m-r \\ r\end{array}\right)\right] B(m-r-1)$.

A nice generalization of the numbers $d_{r}(m, k)$ is given in Theorem 4.1 below. 


\section{Proofs of theorems}

We give the proofs of Theorems 2.1, 2.2, 2.3, 2.7, and 2.8. We recall the basic recurrence for the Stirling set numbers $s 2(m, k)$ [3, page 245], [7]:

$$
\begin{aligned}
& s 2(m, k)=s 2(m-1, k-1)+k s 2(m-1, k), \\
& s 2(0,0)=1, \quad s 2(n, 0)=s 2(0, k)=0 \quad \text { for } n, k>0 .
\end{aligned}
$$

Proof of Theorem 2.1. (i) The set of $t$-separated $k$-partitions of $[m]$ is represented by $H^{t}(m, k)$. To find a member of $H^{t}(m, k)$, we either insert the class $\{m\}$ into any $p \in$ $H^{t}(m-1, k-1)$ in $h^{t}(m-1, k-1)$ possible ways, or put the integer $m$ into any class of each $p \in H^{t}(m-1, k)$ which does not meet the set $\{m-1, m-2, \ldots, m-t+1\}$. Since $\{m-1, m-2, \ldots, m-t+1\}$ has $t-1$ elements which must belong to $t-1$ different classes, the latter case gives rise to $(k-t+1) h^{t}(m-1, k)$ partitions. Thus the result follows:

$$
h^{t}(m, k)=h^{t}(m-1, k-1)+(k-t+1) h^{t}(m-1, k) .
$$

The starting value $h^{t}(m, t)=1$ counts the unique partition of $[m]$ into a complete set of residue classes modulo $t$. The bounds $1 \leq t \leq k \leq m$ are therefore clear.

(ii) We prove (ii) by induction on $m$. The formula is true for $m=1$ since $h^{1}(1,0)=$ $0=s 2(1,0)$, and $h^{1}(1,1)=1=s 2(1,1)$. Assume that (ii) holds for all positive integers up to $m$. Then part (i) gives

$$
\begin{aligned}
h^{t}(m+1, k) & =h^{t}(m, k-1)+(k-t+1) h^{t}(m, k) \\
& =s 2(m-t+1, k-t)+(k-t+1) s 2(m-t+1, k-t+1) \\
& =s 2(m-t, k-t+1)
\end{aligned}
$$

where the second equality is the inductive hypothesis and the third follows from (3.1).

Hence (ii) is true for $m+1$, and the proof is complete.

(iii) This follows from (ii) and the definition of the Bell number $B(m)$ [9, page 20]:

$$
B(m)=\sum_{k} s 2(m, k)
$$

Proof of Theorem 2.2, that is, (2.4). Recall that $C_{r}(m, k)$ is the set of $k$-partitions of $[m]$ with $r$ successions. To find a $p \in C_{r}(m, k)(m>k>0)$, we can either insert the singleton $\{\mathrm{m}\}$ into any $q \in C_{r}(m-1, k-1)$ or we can put the integer $m$ into any $k-1$ classes of a $q \in C_{r}(m-1, k)$, which does not contain $m-1$. There are clearly $(k-1) c_{r}(m-1, k)$ possibilities in the second case. It remains to count those $p \in C_{r}(m, k)$ in which $m$ and $m-1$ belong to the same class. These partitions are obtained by putting $m$ into the class containing $m-1$ in each $q \in C_{r-1}(m-1, k)$. Hence the main result (2.4) follows. The number $c_{0}(m, k)$ is completely determined by the first two cases, that is, $c_{0}(m, k)=c_{0}(m-$ $1, k-1)+(k-1) c_{0}(m-1, k)$, with $c_{0}(1,1)=1, c_{0}(2,1)=0$. Since $c_{0}(m, k)=h^{2}(m+1, k)$, it follows from Theorem 2.1 that $c_{0}(m, k)=s 2(m-1, k-1)$. Since $C_{r}(m, k)$ contains the partition $\{1\}\{2\} \cdots\{k-1\}\{k, k+1, \ldots, m-k+1\}$, and $\{k, k+1, \ldots, m\}$ contains $m-k$ 
successions, we must have $0 \leq r \leq m-k$. The range of $k$ is a consequence of the observation that any $p \in C_{r}(m, k)$ with maximal $k$ has the general form $\{1,2\}\{3,4\} \cdots\{2 r-$ $1,2 r\}\{2 r+1\}\{2 r+2\}, \ldots,\{m\}$.

Proof of Theorem 2.3, that is, (2.5). We apply induction on $m$. The following results show that (2.5) holds for $m=1,2$ :

$$
\begin{aligned}
& c_{0}(1,1)=1=\left(\begin{array}{l}
0 \\
0
\end{array}\right) s 2(0,0), \quad c_{0}(2,1)=0=\left(\begin{array}{l}
1 \\
0
\end{array}\right) s 2(1,0), \quad c_{0}(2,2)=1=\left(\begin{array}{l}
1 \\
0
\end{array}\right) s 2(1,1), \\
& c_{1}(2,1)=1=\left(\begin{array}{l}
1 \\
1
\end{array}\right) s 2(0,0), \quad c_{1}(2,2)=0=\left(\begin{array}{l}
1 \\
1
\end{array}\right) s 2(0,1) .
\end{aligned}
$$

Assume that (2.5) holds for all positive integers up to $m$. Then Theorem 2.2 gives

$$
\begin{aligned}
c_{r}(m+1, k)= & c_{r}(m, k-1)+(k-1) c_{r}(m, k)+c_{r-1}(m, k) \\
= & \left(\begin{array}{c}
m-1 \\
r
\end{array}\right) s 2(m-r-1, k-2)+(k-1)\left(\begin{array}{c}
m-1 \\
r
\end{array}\right) s 2(m-r-1, k-1) \\
& +\left(\begin{array}{c}
m-1 \\
r-1
\end{array}\right) s 2(m-r, k-1) \\
= & \left(\begin{array}{c}
m-1 \\
r
\end{array}\right)(s 2(m-r-1, k-2)+(k-1) s 2(m-r-1, k-1)) \\
& +\left(\begin{array}{c}
m-1 \\
r-1
\end{array}\right) s 2(m-r, k-1) \\
= & \left(\begin{array}{c}
m-1 \\
r
\end{array}\right) s 2(m-r, k-1)+\left(\begin{array}{c}
m-1 \\
r-1
\end{array}\right) s 2(m-r, k-1) \\
= & \left(\begin{array}{c}
m \\
r
\end{array}\right) s 2(m-r, k-1) .
\end{aligned}
$$

The second equality is the inductive hypothesis and the fourth follows from (3.1). Finally, the fifth equality follows from the Pascal triangle of binomial coefficients. Thus (2.5) holds for $m+1$. Hence Theorem 2.3 follows by mathematical induction.

Proof of Theorem 2.7, that is, (2.12). Recall that $D_{r}(m, k)$ denotes the set of $k$-partitions of $[m]$ with $r$-detached successions. There are three ways to locate a member of $D_{r}(m, k)$.

(i) We can insert the singleton $\{\mathrm{m}\}$ into any $p \in D_{r}(m-1, k-1)$ in $d_{r}(m-1, k-1)$ ways.

(ii) We can put the integer $m$ into a class of a $p \in D_{r}(m-1, k)$ which does not contain $m-1$ to get a total of $(k-1)\left(d_{r}(m-1, k)\right)$ partitions.

(iii) Lastly we count the $p \in D_{r}(m, k)$ in which $m$ and $m-1$ belong to a class. These are obtained by putting $m$ into the class containing $m-1$ in any $q \in D_{r-1}(m-1, k)$ in which $m-1$ is not part of a succession. The latter partitions are counted by $d_{r-1}(m-2, k-1)+$ $(k-1) d_{r-1}(m-2, k)$, where $r>0$. Adding all the partitions in (i), (ii), and (iii) gives the 
main result, namely (2.12). For the starting values note that $d_{0}(m, k)=c_{0}(m, k)$ which is $s 2(m-1, k-1)$ by Theorem 2.2. The range of $r$ follows from the fact that $[m]$ contains exactly $\lfloor m / 2\rfloor$ disjoint 2 -subsets of consecutive integers; the range of $k$ is specified as in the proof of Theorem 2.2. The condition on the last inequality follows from the fact that $m<2 r \Rightarrow m-2 r<0 \Rightarrow[m]$ contains less than $r$ distinct pairs of consecutive integers which implies $d_{r}(m, m-r)=0$.

Proof of Theorem 2.8, that is, (2.13). We apply induction on $m$. Since $d_{r}(m, k)=c_{r}(m, k)$ for $r=0,1$, it follows from (the proof of) Theorem 2.3 that (2.13) holds for $m=1,2$. Assume that (2.13) holds for all positive integers up to $m$. Then Theorem 2.7 gives

$$
\begin{aligned}
d_{r}(m+1, k)= & d_{r}(m, k-1)+(k-1) d_{r}(m, k)+d_{r-1}(m-1, k-1)+(k-1) d_{r-1}(m-1, k) \\
= & \left(\begin{array}{c}
m-r \\
r
\end{array}\right) s 2(m-r-1, k-2)+(k-1)\left(\begin{array}{c}
m-r \\
r
\end{array}\right) s 2(m-r-1, k-1) \\
& +\left(\begin{array}{c}
m-r \\
r-1
\end{array}\right) s 2(m-r-1, k-2)+(k-1)\left(\begin{array}{c}
m-r \\
r-1
\end{array}\right) s 2(m-r-1, k-1) \\
= & \left(\begin{array}{c}
m-r \\
r
\end{array}\right)(s 2(m-r-1, k-2)+(k-1) s 2(m-r-1, k-1)) \\
& +\left(\begin{array}{c}
m-r \\
r-1
\end{array}\right)(s 2(m-r-1, k-2)+(k-1) s 2(m-r-1, k-1)) \\
= & \left(\begin{array}{c}
m-r \\
r
\end{array}\right) s 2(m-r, k-1)+\left(\begin{array}{c}
m-r \\
r-1
\end{array}\right) s 2(m-r, k-1) \\
= & \left(\begin{array}{c}
m-r+1 \\
r-1
\end{array}\right) s 2(m-r, k-1) .
\end{aligned}
$$

The second equality is the inductive hypothesis, the fourth follows from (3.1), and the last follows from the Pascal triangle of binomial coefficients. Hence the proof of Theorem 2.8 follows by mathematical induction.

\section{Some generalizations}

Let a partition of $[m]$ be said to have $r$ t-successions $(r \geq 0, t \geq 1)$ if it contains exactly $r t$-strings of consecutive integers, where each $t$-string of consecutive integers is counted within one class, and a string of more than $t$ consecutive integers in a class are considered $t$ at a time. Denote the set of $k$-partitions of $[m]$ with $r t$-successions by $C_{t}(m, k, r)$. As before let $c_{t}(m, k, r)=\left|C_{t}(m, k, r)\right|$. It follows that $c_{r}(m, k)=c_{2}(m, k, r)$ and $c_{0}(m, k)=$ $c_{1}(m, k, m)$. Similarly, we generalize $D_{r}(m, k)$ by letting $D_{t}(m, k, r)$ represent the set of $k$-partitions of $[m]$ with $r t$-successions in which every string of consecutive integers appearing in a class has length 1 or $t$. Thus $D_{r}(m, k)=D_{2}(m, k, r) ; d_{t}(m, k, r)=\left|D_{t}(m, k, r)\right|$.

It turns out that there is an easy closed formula for $d_{t}(m, k, r)$ whereas the one for $c_{t}(m, k, r)$ remains inscrutable.

The proofs of the first two parts of the following theorem are analogous to those of Theorems 2.7, 2.8, respectively. The details are omitted. 
Theorem 4.1. (i) $d_{t}(m, k, r)=d_{t}(m-1, k-1, r)+(k-1) d_{t}(m-1, k, r)+d_{t}(m-t, k-$ $1, r-1)+(k-1) d_{t}(m-t, k, r-1), 0 \leq r \leq[m / t], 1 \leq k \leq m-(t-1) r, d_{t}(m, k, 0)=$ $s 2(m-1, k-1)$.

(ii) $d_{t}(m, k, r)=\left(\begin{array}{c}m-(t-1) r \\ r\end{array}\right) s 2(m-(t-1) r-1, k-1)$.

(iii) $d_{t}(m, r)=\sum_{k} d_{t}(m, k, r)=(\underset{r}{m-(t-1) r}) B(m-(t-1) r-1)$.

It follows from Theorem 4.1(ii) that

$$
\begin{aligned}
d_{t}(m, k, r) & =d_{t-j}(m-j r, k, r), \quad j=0,1, \ldots, t-1, \\
& =d_{1}(m-(t-1) r, k, r)=\left(\begin{array}{c}
m-(t-1) r \\
r
\end{array}\right) d_{0}(m-(t-1) r, k),
\end{aligned}
$$

where $d_{1}(m, k, r)=\left(\begin{array}{c}m \\ r\end{array}\right) d_{1}(m, k, m), d_{0}(m, k)=d_{1}(m, k, m)$.

Let $W_{t}(m, k, r)$ represent the set of $k$-partitions of [ $\left.m\right]$ with $r t$-successions in which every string of consecutive integers appearing in a class has length at most $t$. Then the set difference $E_{t}(m, k, r)=C_{t}(m, k, r)-W_{t}(m, k, r)$ consists of those partitions in $C_{t}(m, k, r)$ in which at least one class in each partition contains a string of $t+1$ consecutive integers, provided that both $t$ and $r$ are greater than 1. It follows that $D_{t}(m, k, r) \subseteq W_{t}(m, k, r) \subseteq$ $C_{t}(m, k, r)$, with $D_{2}(m, k, r)=W_{2}(m, k, r)$, and $W_{t}(m, k, r)=C_{t}(m, k, r)$ for $(t, r)=(1, r)$, $(t, 1)$. As usual let $w_{t}(m, k, r)=\left|W_{t}(m, k, r)\right|$.

We are unable to find a concise formula for $w_{t}(m, k, r)(t>2)$ (and hence $c_{t}(m, k, r)$ ), not even when $t=3$ and $r=1$. However, we have the following computational result which is established by extending the inclusion-exclusion-type reasoning used in the proof of Theorem 2.2.

Corollary 4.2. $w_{t}(m, k, r)$ satisfies the following recurrence:

$$
\begin{aligned}
w_{t}(m, k, r)= & w_{t}(m-1, k-1, r)+(k-1) w_{t}(m-1, k, r)+w_{t}(m-t, k-1, r-1) \\
& +(k-1) w_{t}(m-t, k, r-1)+\sum_{u=1}^{t-2}\left(w_{t}(m-u-1, k-1, r)\right. \\
& \left.+(k-1) w_{t}(m-u-1, k, r)\right),
\end{aligned}
$$

$0 \leq r \leq[m / t], 1 \leq k \leq m-(t-1) r, w_{t}(m, k, 0)=\sum_{j=0}^{\lfloor m /(t-1)\rfloor} w_{t-1}(m, k, j)$, where $\lfloor N\rfloor$ is the integer part of $N$.

Proof. There are three ways to find an element of $W_{t}(m, k, r)$.

(1) We can insert the singleton $\{\mathrm{m}\}$ into any $p \in W_{t}(m-1, k-1, r)$ in $w_{t}(m-1, k-$ $1, r)$ possible ways.

(2) We can put the integer $m$ into a class of $p \in W_{t}(m-1, k, r)$ under two situations:

(i) if $m-1$ is part of a $v$-succession, $t-1 \leq v \leq t(k \geq 2)$, then put $m$ into any class of $p$ which does not contain $m-1$;

(ii) else put $m$ into any class of $p$.

We note that (ii) requires only those $p \in W_{t}(m-1, k, r)$ in which the integer $m-$ 1 is part of a $u$-succession for $1 \leq u \leq t-2$. Thus the total number of partitions 
from (ii) is

$$
k \sum_{u=1}^{t-2}\left(w_{t}(m-u-1, k-1, r)+(k-1) w_{t}(m-u-1, k, r)\right)
$$

since $w_{t}(m-u-1, k-1, r)+(k-1) w_{t}(m-u-1, k, r)$ counts the $p \in W_{t}(m-$ $1, k, r)$ in which $m-1$ is part of a $u$-succession: treating the $u$ numbers $m-u, m-$ $u+1, \ldots, m-1$ as an integer $N$, then a needed $p \in W_{t}(m-1, k, r)$ can be formed by inserting $\{N\}$ into a $q \in W_{t}(m-u-1, k-1, r)$ in $w_{t}(m-u, k-1, r)$ possible ways, or by putting $N$ into any of $k-1$ classes of each $q \in W_{t}(m-u-1, k, r)$ which does not contain $m-u-1$, which can happen in $(k-1) w_{t}(m-u-1, k, r)$ ways. Thus the total number of partitions from (i) is

$$
(k-1)\left(w_{t}(m-1, k, r)-\sum_{u=1}^{t-2}\left(w_{t}(m-u-1, k-1, r)+(k-1) w_{t}(m-u-1, k, r)\right)\right) .
$$

Hence the number of partitions from (2.5), by adding (4.3) and (4.4), is

$$
(k-1) w_{t}(m-1, k, r)+\sum_{u=1}^{t-2}\left(w_{t}(m-u-1, k-1, r)+(k-1) w_{t}(m-u-1, k, r)\right) .
$$

(3) We count the $p \in W_{t}(m, k, r)$ in which $m$ and $m-1$ belong to a $t$-succession. These are obtained by putting $m$ into the class of $m-1$ in any $q \in W_{t}(m-1, k, r-$ 1) containing $m-1$ as part of a $(t-1)$-succession. The required partitions are enumerated by

$$
w_{t}(m-t, k-1, r-1)+(k-1) w_{t}(m-t, k, r-1) .
$$

Adding all the partitions from (1), (2), and (3) gives the desired result:

$$
\begin{aligned}
w_{t}(m, k, r)= & w_{t}(m-1, k-1, r)+(k-1) w_{t}(m-1, k, r) \\
& +\sum_{u=1}^{t-2}\left(w_{t}(m-u-1, k-1, r)+(k-1) w_{t}(m-u-1, k, r)\right) \\
& +w_{t}(m-t, k-1, r-1)+(k-1) w_{t}(m-t, k, r-1) .
\end{aligned}
$$

It is clear that $w_{t}(m, k, 0)=\sum_{j=0}^{\lfloor m /(t-1)\rfloor} w_{t-1}(m, k, j)$. This completes the proof of Corollary 4.2 .

Remark 4.3. In the proof of Corollary 4.2, the apparent simplification suggested by enumerating the partitions from 2(i) first (since there are only two summands), and then obtaining those from 2 (ii) by complementation, leads to the following three-group contribution instead of (4.5):

$$
k w_{t}(m-1, k, r)-\sum_{v=t-1}^{t}\left(w_{t}(m-v-1, k-1, r)+(k-1) w_{t}(m-v-1, k, r)\right) .
$$


However, (4.8) will not always give correct results because it is inconsistent with the cumulative "origin" $w_{t}(m, k, 0)$. This is easily verified by a few actual computations.

Corollary 4.2 is a special case of the following result.

Theorem 4.4. $c_{t}(m, k, r)$ satisfies the following recurrence:

$$
\begin{aligned}
c_{t}(m, k, r)= & c_{t}(m-1, k-1, r)+(k-1) c_{t}(m-1, k, r)+c_{t}(m-1, k, r-1) \\
& +\sum_{u=1}^{t-2}\left(c_{t}(m-u-1, k-1, r)+(k-1) c_{t}(m-u-1, k, r)\right) \\
& -\sum_{u=1}^{t-2}\left(c_{t}(m-u-1, k-1, r-1)+(k-1) c_{t}(m-u-1, k, r-1)\right), \\
c_{t}(m, k, 0)= & \sum_{j=0}^{\lfloor m /(t-1)\rfloor} w_{t-1}(m, k, j), \\
& 0 \leq r \leq m-k-t+2, \quad 1 \leq t \leq m, \quad 1 \leq k \leq m-r(t-1) .
\end{aligned}
$$

Before sketching the proof of Theorem 4.4 we state the special case of $t=3$.

Corollary 4.5 .

$$
\begin{aligned}
c_{3}(m, k, r)= & c_{3}(m-1, k-1, r)+(k-1) c_{3}(m-1, k, r)+c_{3}(m-2, k-1, r) \\
& +(k-1) c_{3}(m-2, k, r)+c_{3}(m-1, k, r-1)-c_{3}(m-2, k-1, r-1) \\
& -(k-1) c_{3}(m-2, k, r-1), \\
c_{3}(m, k, 0)= & \sum_{j=0}^{\lfloor m / 2\rfloor}\left(\begin{array}{c}
m-j \\
j
\end{array}\right) s 2(m-1-j, k-1), \\
& 0 \leq r \leq m-k-1, \quad 1 \leq k \leq m-2 r .
\end{aligned}
$$

(A derivation of the pool of starting values $c_{3}(m, k, 0)$ is given at the end of the proof of Theorem 4.4 below.)

Using (4.10) the numbers $c_{3}(m, k, r)$ are computed and displayed in Tables 4.1 and 4.2 for $r=1$ and $r=2$, where $1 \leq m \leq 10$ and $1 \leq k \leq m$. The row sums $c_{3}(m, r)=$ $\sum_{k} c_{3}(m, k, r)$ are given in the last columns.

Proof of Theorem 4.4. The contributions to $C_{t}(m, k, r)$ follow exactly as in the proof of Corollary 4.2 except in the third case, that is,

(1) $c_{t}(m-1, k-1, r)$;

(2) $(k-1) c_{t}(m-1, k, r)+\sum_{u=1}^{t-2}\left(c_{t}(m-u-1, k-1, r)+(k-1) c_{t}(m-u-1, k, r)\right)$;

(3) this time, the $p \in C_{t}(m, k, r)$ in which $m$ and $m-1$ belong to a $t$-succession are obtained by putting $m$ into the class of $m-1$ in the $q \in C_{t}(m-1, k, r-1)$ having $m-1$ as part of a $v$-succession, where $t-1 \leq v \leq t+r-1$. By part (2)(i) 
462 Set partitions with successions and separations

Table 4.1. Partitions of $[m]$ with one three-succession $c_{3}(m, k, 1)$.

\begin{tabular}{rrrrrrrrrrr}
\hline$m \backslash k$ & 1 & 2 & 3 & 4 & 5 & 6 & 7 & 8 & 9 & Sum \\
\hline 1 & 0 & - & - & - & - & - & - & - & - & 0 \\
2 & 0 & 0 & - & - & - & - & - & - & - & 0 \\
3 & 1 & 0 & 0 & - & - & - & - & - & - & 1 \\
4 & 0 & 2 & 0 & 0 & - & - & - & - & - & 2 \\
5 & 0 & 5 & 3 & 0 & 0 & - & - & - & - & 8 \\
6 & 0 & 10 & 18 & 4 & 0 & 0 & - & - & - & 32 \\
7 & 0 & 20 & 74 & 42 & 5 & 0 & 0 & - & - & 141 \\
8 & 0 & 38 & 266 & 282 & 80 & 6 & 0 & 0 & - & 672 \\
9 & 0 & 71 & 889 & 1564 & 785 & 135 & 7 & 0 & 0 & 3451 \\
10 & 0 & 130 & 2846 & 7808 & 6150 & 1810 & 210 & 8 & 0 & 18962 \\
\hline
\end{tabular}

Table 4.2. Partitions of $[m]$ with two three-successions $c_{3}(m, k, 2)$.

\begin{tabular}{rrrrrrrrrrrr}
\hline$m \backslash k$ & 1 & 2 & 3 & 4 & 5 & 6 & 7 & 8 & 9 & 10 & Sum \\
\hline 1 & 0 & - & - & - & - & - & - & - & - & - & 0 \\
2 & 0 & 0 & - & - & - & - & - & - & - & - & 0 \\
3 & 0 & 0 & 0 & - & - & - & - & - & - & - & 0 \\
4 & 1 & 0 & 0 & - & - & - & - & - & - & - & 1 \\
5 & 0 & 2 & 0 & 0 & 0 & - & - & - & - & - & 2 \\
6 & 0 & 6 & 3 & 0 & 0 & 0 & - & - & - & - & 9 \\
7 & 0 & 13 & 21 & 4 & 0 & 0 & 0 & - & - & - & 38 \\
8 & 0 & 29 & 95 & 48 & 5 & 0 & 0 & 0 & - & - & 177 \\
9 & 0 & 60 & 372 & 354 & 90 & 6 & 0 & 0 & 0 & - & 882 \\
10 & 0 & 122 & 1342 & 2125 & 965 & 150 & 7 & 0 & 0 & 0 & 4711 \\
\hline
\end{tabular}

the number of such partitions is

$$
c_{t}(m-1, k, r-1)-\sum_{u=1}^{t-2}\left(c_{t}(m-u-1, k-1, r-1)+(k-1) c_{t}(m-u-1, k, r-1)\right) .
$$

Adding all the partitions from (1), (2), and (3) gives the desired result:

$$
\begin{aligned}
c_{t}(m, k, r)= & c_{t}(m-1, k-1, r)+(k-1) c_{t}(m-1, k, r) \\
& +\sum_{u=1}^{t-2}\left(c_{t}(m-u-1, k-1, r)+(k-1) c_{t}(m-u-1, k, r)\right)+c_{t}(m-1, k, r-1) \\
& -\sum_{u=1}^{t-2}\left(c_{t}(m-u-1, k-1, r-1)+(k-1) c_{t}(m-u-1, k, r-1)\right) .
\end{aligned}
$$


For starting values, we have $c_{t}(m, k, 0)=\sum_{j=0}^{\lfloor m /(t-1)\rfloor} w_{t-1}(m, k, j)$ since a partition without a $t$-succession has successions of length at most $t-1$. In particular if $t=3$, then $c_{3}(m, k, 0)=\sum_{j=0}^{\lfloor m / 2\rfloor} w_{2}(m, k, j)$, which equals $\sum_{j=0}^{\lfloor m / 2\rfloor}\left(\begin{array}{c}m-j \\ j\end{array}\right) s 2(m-1-j, k-1)$, by Theorem 2.3. This completes the proof of Theorem 4.4 .

\section{References}

[1] G. E. Andrews, The Theory of Partitions, Addison-Wesley Publishing, Massachusetts, 1976, reprinted by Cambridge University Press, Cambridge in 1984 and 1998.

[2] C. C. Chen and K. M. Koh, Principles and Techniques in Combinatorics, World Scientific Publishing, New Jersey, 1992.

[3] R. L. Graham, D. E. Knuth, and O. Patashnik, Concrete Mathematics.A Foundation for Computer Science., Addison-Wesley Publishing, Massachusetts, 1989.

[4] A. O. Munagi, $k$-complementing subsets of nonnegative integers, Int. J. Math. Math. Sci. 2005 (2005), no. 2, 215-224.

[5] J. Riordan, An Introduction to Combinatorial Analysis, Wiley Publications in Mathematical Statistics, John Wiley \& Sons, New York, 1958.

[6] Combinatorial Identities, John Wiley \& Sons, New York, 1968.

[7] E. W. Weisstein, Stirling Number of the Second Kind, From MathWorld-A Wolfram Web Resource, http://mathworld.wolfram.com/StirlingNumberoftheSecondKind.html.

[8] H. S. Wilf, East side, west side — an introduction to combinatorial families with Maple programming, preprint, 2002.

[9] _ Generatingfunctionology, 2nd ed., Academic Press Inc., San Diego, 1994.

Augustine O. Munagi: Department of Mathematics, University of Lagos, Akoka-Yaba, Lagos, Nigeria

E-mail address: amunagi@yahoo.com 


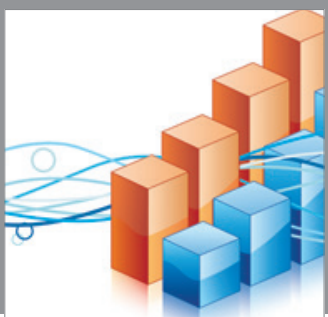

Advances in

Operations Research

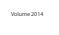

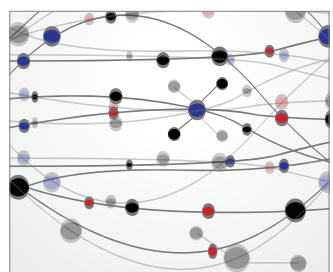

\section{The Scientific} World Journal
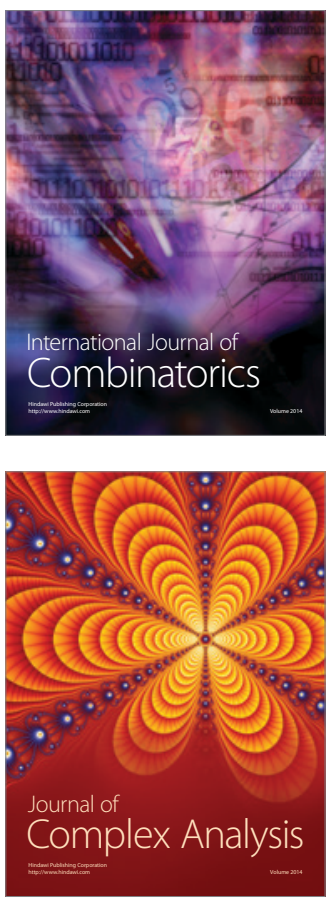

International Journal of

Mathematics and

Mathematical

Sciences
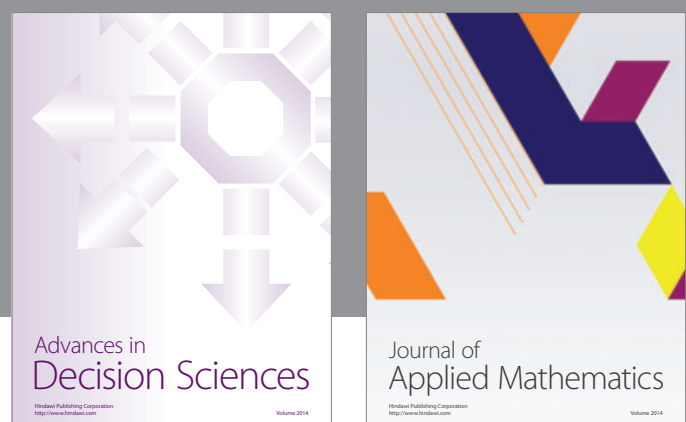

Journal of

Applied Mathematics
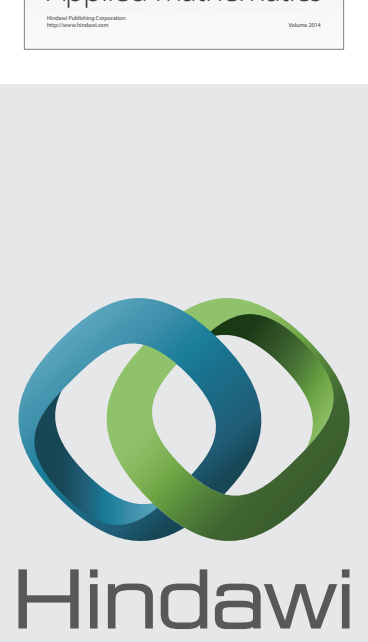

Submit your manuscripts at http://www.hindawi.com
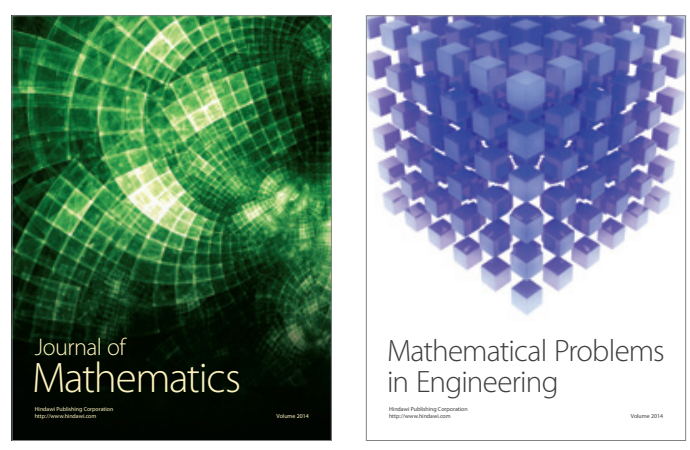

Mathematical Problems in Engineering
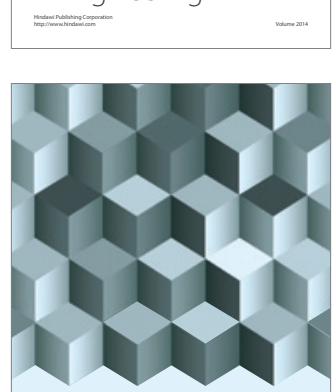

Journal of

Function Spaces
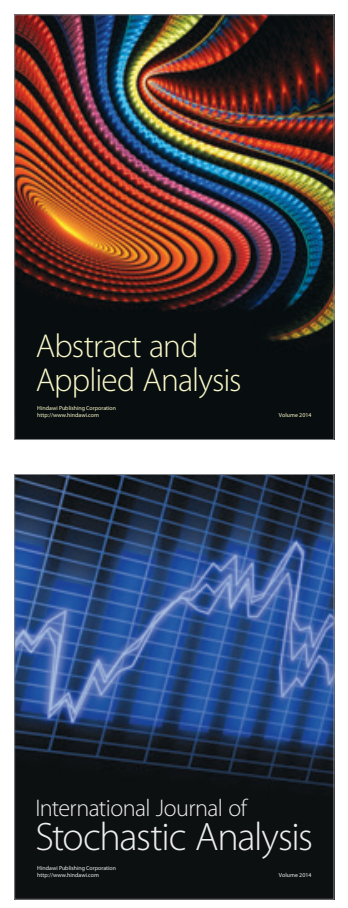

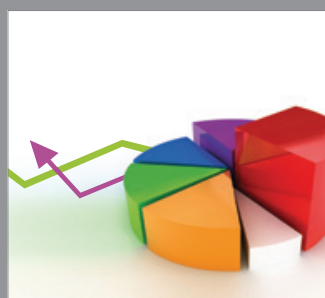

ournal of

Probability and Statistics

Promensencen
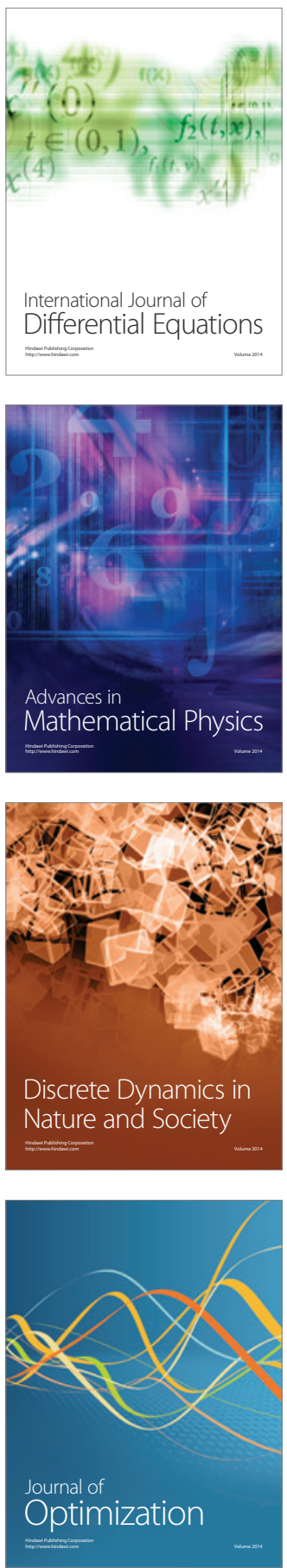